ARTÍCULO ORIGINAL
REVISTA CDLIMBIANA
DE CIENCIAANIMAL

Rev Colombiana Cienc Anim 2016; 8(2):219-223.

\title{
Habronemosis peneana en un burro criollo (Equus asinus) del departamento de Sucre
}

\section{Penile habronemosis in a creole donkey (Equus asinus) in Sucre department}

\author{
CARDONA A JOSÉ ${ }^{1 *}$ Ph.D, VIOLET L LINA² MVZ, \\ MONTES $\vee$ DONICER ${ }^{3}$ Ph.D.
}

${ }^{1}$ Universidad de Córdoba, Departamento de Ciencias Pecuarias, Grupo de Investigaciones en Medicina de Grandes Animales (MEGA), Montería, Colombia. 2Universidad de Córdoba, Facultad de Medicina Veterinaria y Zootecnia, Departamento de Ciencias Pecuarias, Montería, Colombia. ${ }^{3}$ Universidad de Sucre ,Facultad de Ciencias Agropecuarias, Departamento de Zootecnia, Sincelejo, Colombia.

Keywords:

Dermopathies, diagnostic, equine, histopathology.

Palabras Clave:

Dermopatías, diagnóstico, equino, histopatología.

\section{Abstract}

The aim of this study was to evaluate the clinical and histopathologic characteristics of a case of penile Habronemosis in a Creole donkey (Equus asinus), whole male, 5 years old and $160 \mathrm{~kg}$, from the municipality of Coloso (Sucre, Colombia ). Was performed clinical evaluation and histopathological characterization of the affected area, finding the presence of a lesion fast, granulomatous, exophytic growth, crater-shaped and reddish at the level of the glans. Later, after sedation and application of local anesthesia it was taken a biopsy of tissue from the periphery of the granulomatous lesion with a punch of $6 \mathrm{~mm}$, fixed in $10 \%$ formalin and taken to the laboratory of pathology, where they were processed to inclusion in paraffin. Finally, the sample was stained with hematoxylin-eosin (HE), where the presence of active pyogranulomatous dermatitis was confirmed, with areas of diffuse multifocal necrosis, with marked presence of eosinophils and neutrophils, as well as the phenomenon of Splendore-Hoeppli, corresponding sites larvae location surrounded by a dense shell eosinofilica. The definitive diagnosis was Habronemosis, and despite being a frequent reason for consultation in the veterinary clinic, this is the first report described in donkeys Colombia.

\section{Resumen}

El objetivo de este estudio fue evaluar las características clínicas e histopatológicas de un caso de Habronemosis peneana en un burro criollo (Equus asinus), macho entero, de 5 años de edad y $160 \mathrm{~kg}$ de peso, procedente del municipio de Coloso (Sucre, Colombia). Se le realizó evaluación clínica y caracterización anatomopatológica del área afectada, encontrándose presencia de una lesión de crecimiento rápido, granulomatosa, exofítica, en forma de cráter y de coloración rojiza a nivel del glande. Posteriormente, previa sedación y aplicación de anestesia local fue tomada una biopsia de tejido desde la periferia de la lesión granulomatosa con punch de $6 \mathrm{~mm}$, fijada en formol al 10\% y llevadas al laboratorio de patología, Colombia, donde fueron procesadas hasta su inclusión en parafina. Finalmente, la muestra fue teñida con la coloración de Hematoxilina-Eosina ( $\mathrm{H}-\mathrm{E})$, donde se ratificó la presencia de dermatitis piogranulomatosa activa, conáreas de necrosis multifocal difusas, con marcada presencia de eosinófilos y neutrófilos, así como el fenómeno de Splendore-Hoeppli, que corresponde sitios de ubicación de larvas rodeadas por una envoltura eosinofílica densa. El diagnóstico definitivo fue de Habronemosis, y a pesar de ser un motivo frecuente de consulta en la clínica veterinaria, este es el primer reporte descrito en burros de Colombia. 


\section{Introducción}

La Habronemosis, es una enfermedad parasitaria de distribución mundial que afecta a los equinos, producida por los nematodos (Orden: Spirurida), Habronema muscae, Habronema majus $(H$. microtoma), y Draschia megastoma (PUGH et al., 2014). En su estado adulto se les encuentra en el estómago de los equinos, por lo que son llamados gusanos del estómago (MEHLHORN et al., 2008); los huevos que contienen la larva L1 viajan a través del tracto gastrointestinal y son eliminados en las heces, donde serán ingeridos por larvas (L3) de moscas, una vez ingeridas, las larvas de Habronema maduran y se desarrollan dentro de la puppa hasta llegaran al estado infectivo (L3); cuando la mosca eclosiona, la larva (L3) de habronema migra hacia la probóscide del díptero, el cual al posarse sobre la boca del equino, las deposita y estas viajan a través del esófago para después ser ingerida por el animal y así llegar al estómago, completando el ciclo (SCHUTER et al., 2010; AL ANAZI et al., 2011; BUZZELL et al., 2011). Las especies de mosca involucradas en este ciclo son principalmente Musca domestica y Stomoxys calcitrans, aunque pueden servir de huéspedes intermediarios otras especies de moscas como Fannia sp, Haematobia irritans, M. autumnalis, Musca humilis, M. insoria, M. terrareginae, M. hilli, pseudopyrellia spp, y Sarcophaga misera (SCHUTER et al., 2010; PUGH et al., 2014).

Las moscas tienen preferencia por cualquier tipo de tejido blando, mucosas, secreciones no profusas y material orgánico del cual alimentarse, por lo que no se posan solo en la boca sino que también pueden posarse sobre los orificios nasales, conjuntiva y heridas abiertas; haciendo que se presenten infecciones por larvas erráticas de habronema en pulmón, conjuntiva y piel respectivamente, esta última en zonas predispuestas a traumas como miembros, genitales y abdomen (PUGH et al., 2014).

A nivel gástrico, $H$. majus y $H$. muscae causan poco o ningún cambio patológico en el estómago equino, Sin embargo, una gran cantidad de parásitos pueden causar gastritis catarral y/o ulcerosa; en especial $D$. megastoma, la cual puede producir masas granulomatosas de hasta $10 \mathrm{~cm}$ de diámetro, compuestos de gusanos adultos y el material necrótico; estas lesiones se presentan en rara ocasión, pero pueden presentarse obstrucciones a nivel de píloro y producir una perforación de estómago, que puede conducir a una peritonitis (PUGH et al.,2014).
Histopatológicamente, se ha reportado infiltración difusa de células inflamatorias mononucleares con un número de eosinófílos en la mucosa y submucosa del estómago que sugerían gastritis eosinofílica (AMININAJAFI et al., 2014) En piel, las lesiones son de tipo granulomatosas en forma de cráter y exofíticas (DOWN et al., 2009), con crecimiento rápido y coloración marrón rojiza, en algunos casos, con larvas amarilla calcificadas cuya apariencia es similar a un grano de arroz, así mismo, en la histopatología la epidermis se observa ulceradas con una fina capa de restos celulares necróticos mezclados con neutrófilos. La dermis y el tejido subcutáneo se observan marcadamente congestivo, edematoso y con extensos infiltrados de células inflamatorias, predominantemente eosinófilos con menor número de células plasmáticas, macrófagos, y linfocitos (AMININAJAFI et al., 2014; PUGH et al., 2014).

El diagnostico está basado en los antecedentes clínicos, las características clínicas de la lesión y su respectiva biopsia, la cual al ser teñida con hematoxilina eosina presenta áreas de necrosis multifocal difusas y marcada eosinofília, indicando dermatitis piogranulomatosa activa, en algunos casos se puede observar las larvas rodeadas por una envoltura eosinofílica densa (DOWN et al., 2009; FREITAS, 2013; PUGH et al., 2014), correspondiente al fenómeno de SplendoreHoeppli (SH).

Se ha reportado tratamiento generalmente usando avermectinas y milbemicinas, así como sus análogos estructurales, pomadas preparadas con antiinflamatorios, Triclorón, Coumafos (PATERSON, 2009; ARRIETA et al.; 2013) antibióticos, así como la extirpación quirúrgica con posterior aplicación de nitrógeno líquido (TRAVERSA et al.; 2007; PUGH et al.; 2014). El objetivo de este estudio fue evaluar las características clínicas e histopatológicas de un caso de Habronemosis peneana en un burro criollo (Equus asinus) del departamento de Córdoba, Colombia.

\section{Materiales y métodos}

En el mes de mayo del año 2014, fue atendido por el servicio clínico ambulatorio de grandes animales de la Facultad de Medicina Veterinaria y Zootecnia de la Universidad de Córdoba, un burro (Equus asinus), criollo, macho entero, de 5 años de edad y $160 \mathrm{~kg}$ de peso, procedente del municipio de Coloso (Sucre, Colombia), al cual se le realizo examen clínico general, dermatológico 
y reproductivo especializado, consistente en evaluación de la región inguinal y peneana comprometida, la cual presentó una lesión de crecimiento rápido, granulomatosa, exofítica, en forma de cráter y de coloración rojiza a nivel del glande (Figura 1) y que a la compresión presentó por algunos sitios de la lesión, salida de estructuras calcificadas amarillas cuya apariencia era similar a un grano de arroz, que posiblemente correspondían a larvas de parásitos (Figura 2) compatibles con Habronemosis.
Posteriormente, previa sedación (Xilacina 10\%, Erma ${ }^{\circledR}$, Colombia) y aplicación de anestesia local (Lidocaina $2 \%$, Synthesis $®$, Colombia) fue tomada una biopsia de tejido desde la periferia de la lesión granulomatosa (CARDONA et al.; 2014), con punch de $6 \mathrm{~mm}$, posteriormente fijada en formol al $10 \%$ y llevadas al laboratorio de Patología del Departamento de Ciencias Pecuarias de la Universidad de Córdoba, Colombia, donde fueron procesadas hasta su inclusión en parafina. Posteriormente, la muestra fue teñida con la coloración de Hematoxilina Eosina $(\mathrm{H}-\mathrm{E})$, para confirmar la presencia de dermatitis piogranulomatosa activa.

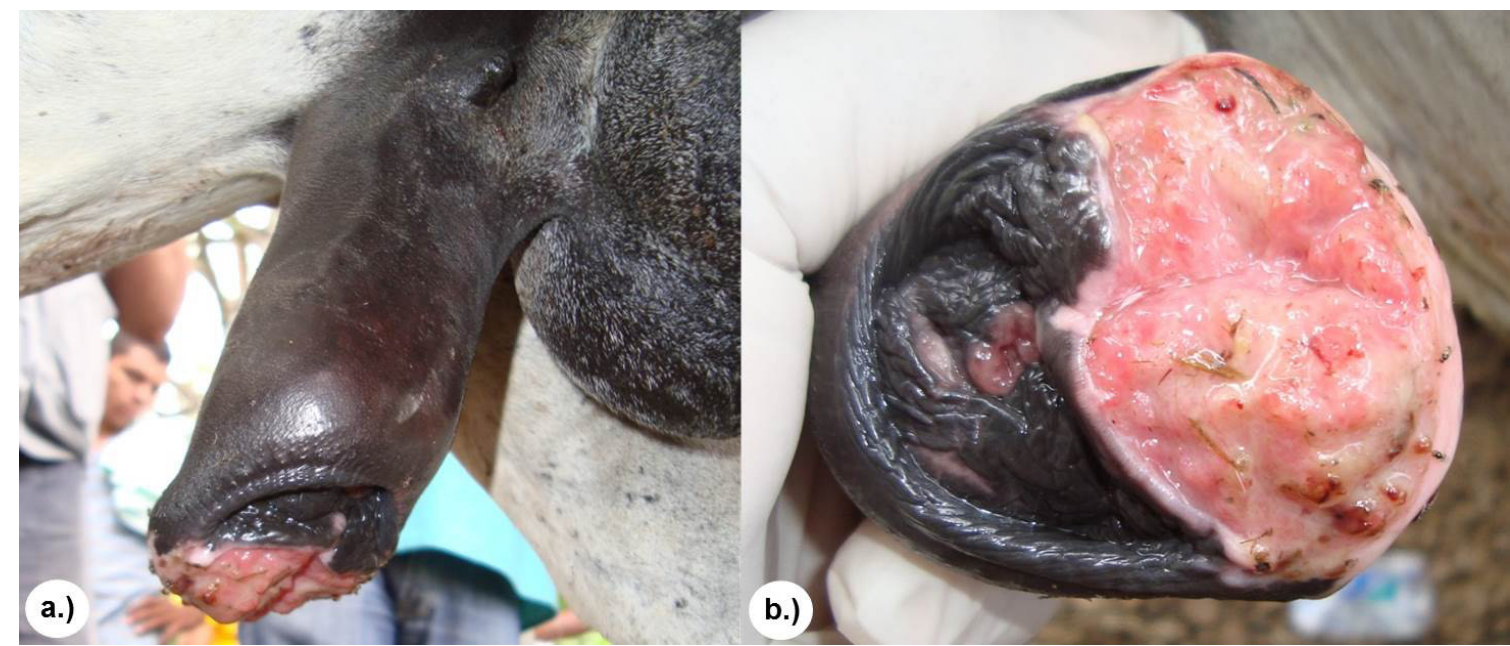

Figura 1. Habronemosis equina. a.) Ubicación a nivel de glande. b.) Lesión granulomatosa, exofítica, en forma de cráter y de coloración rojiza.

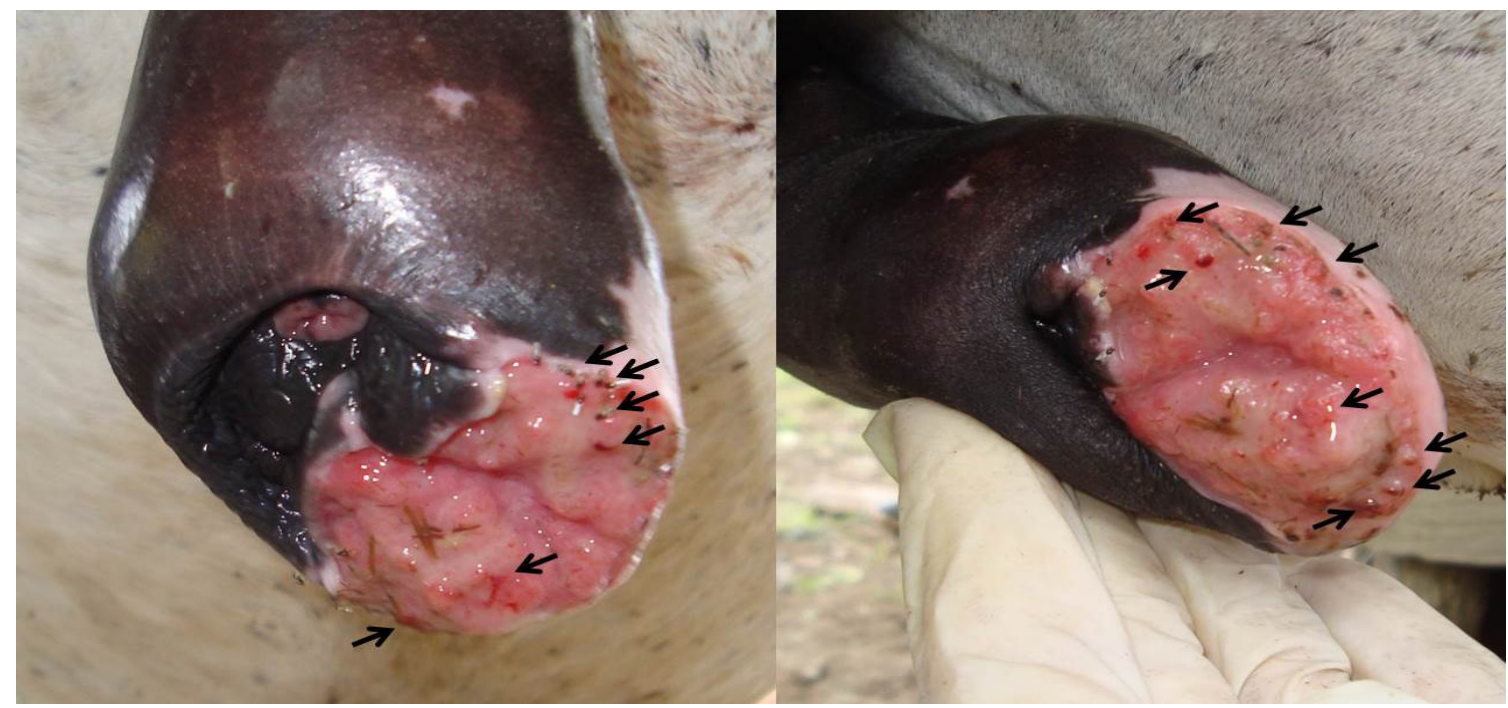

Figura 2. Lesión granulomatosa a nivel de glande con puntos de salida de material compatible con larvas calcificadas (flechas). 


\section{Resultados y discusión}

Los datos de la anamnesis y la historia clínica, así como las manifestaciones obtenidas en la exploración física, las características anatomopatológicas de la lesión y los resultados histopatológicos (Fig 3), confirmaron el diagnóstico de habronemosis peneana en un burro criollo, estudiado de acuerdo con lo reportado como método diagnóstico de la condición patológica (DOWN et al., 2009; FREITAS, 2013; PUGH et al., 2014).

El corte histopatológico denotó la presencia de dermatitis piogranulomatosa activa, observándose áreas de necrosis multifocal difusas, con marcada presencia de eosinófilos y neutrófilos, así como el fenómeno de Splendore-Hoeppli, que corresponde sitios de ubicación de larvas rodeadas por una envoltura eosinofíilica densa (Figura 3 ). Las características histopatológicas obtenidas al teñir la muestra con Hematoxilina-Eosina (H-E), corresponden a las reportadas por DOWN et al. 2009; SCHUSTER et al. 2010; AMININAJAFI et al. 2014; en las que se describe una dermatitis granulomatosa, con diferentes áreas de necrosis y gran número de eosinófilos en la periferia.

La ubicación anatómica de la lesión del animal estudiado corresponde a unos de los sitios en el que se presenta con mayor frecuencia la Habronemosis cutánea (PUGH et al., 2014), al parecer por ser una zona húmeda con constante secreción no profusa de moco y estar propensa a traumas previos durante la monta. Macroscópicamente presenta una única lesión, aunque DOWN et al. (2009) señalan que tambien se pueden observar múltiples lesiones en un mimso individuo; Las carateriscticas son similares a las descritas por DOWN et al. (2009) y PUGH et al. (2014), siendo de apariencia granulomatosa, exofítica, en forma de cráter y de coloración rojiza; con presencia de material calcificado amarillo similar a un grano de arroz.

La fecha en la que se presentó el caso coincide con el inicio de la época de lluvias, en esta época se observa con frecuencia la acumulación y mal manejo del estiércol, pienso y materia orgánica en descomposición, así como paja y camas húmedas, situación favorece la reproducción y posterior proliferación de moscas (PUGH et al., 2014). Coincidiendo con lo reportado por TRAVERSA et al. (2007), donde se señala que este tipo de lesiones se presentan con mayor frecuencia en las épocas de alta actividad de moscas.

A pesar de ser un motivo frecuente de consulta en la clínica veterinaria, este es el primer reporte descrito en Colombia, por lo que es necesario continuar con investigaciones sobre factores de riesgo, incidencia y prevalencia, debido al potencial de esta enfermedad para generar impacto negativo en la salud y aprovechamiento económico de los equinos.

Basado en el diagnóstico clínico y por ser un semoviente reproductor, con el fin de prolongar la vida reproductiva del animal, se realizó tratamiento médico basado en antihelmínticos tipo ivermectina $1 \%$ a dosis de $0,2 \mathrm{mg} / \mathrm{kg} / \mathrm{im}$ y aplicación tópica de pomada a base de triclorfón.

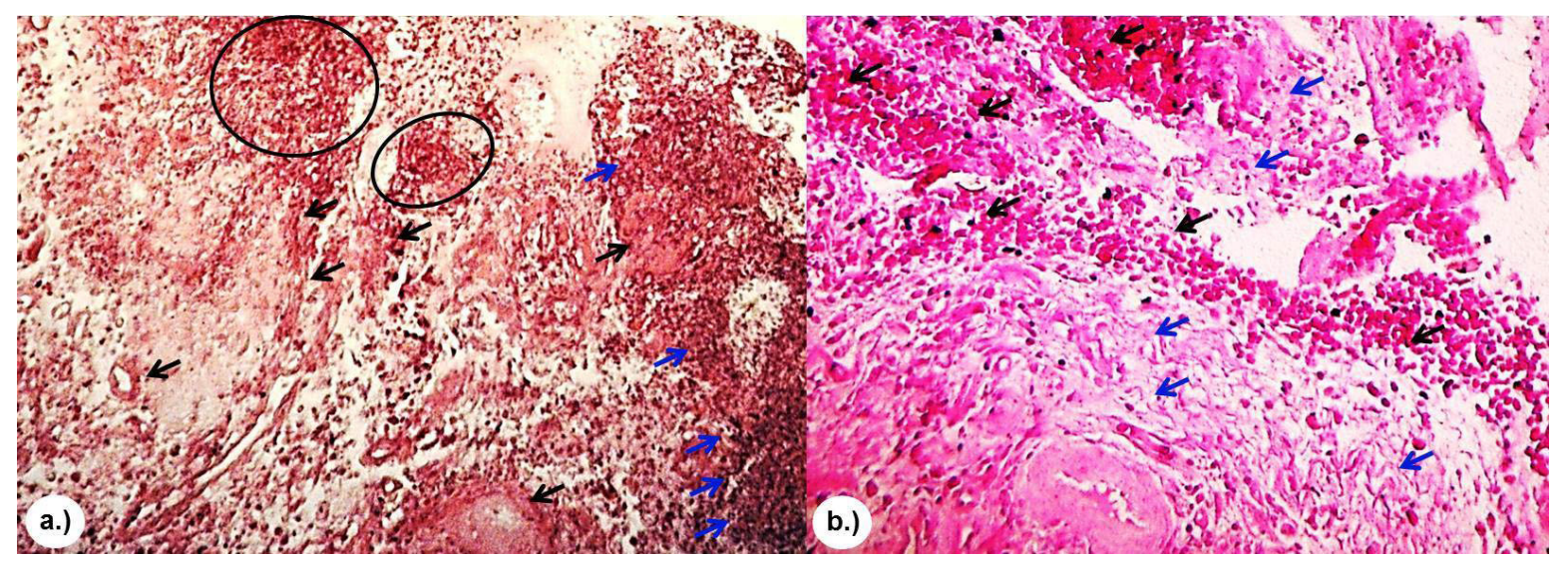

Figura 3. Tinción de H-E. a.) Severa dermatitis piogranulomatosa, obsérvese la presencia de múltiples fenómenos de Splendore-Hoeppli (flechas negras) con focos de eosinófilos y neutrófilos (círculos) y áreas de marcada necrosis (flechas azules). 20X. b.) Áreas de edema (flechas azules) y congestión (flechas negras). 20X. 


\section{Referencias}

AL ANAZI, A.; ALYOUSIF, M. 2011. Prevalence of non-strongyle gastrointestinal parasites of horses in Riyadh region of Saudi Arabia. Saudi J Biol Sci 18(3): 299-303.

ARRIETA, R.; FERNÁNDEZ, JOSÉ.; MONTALVO, E. 2013. Habronemosis en potranca: informe de caso clínico. Redvet 14(11): 1-5.

AMININAJAFI, F.; MEHRARA, M.; HOSSEINI, A.; FATTAHI, R.; TAGHIZADEH, M.; HASANZADEH, S. 2014. Histopathological features of cutaneous and gastric habronemiasis in horse. J Parasit Dis.

Buzzell, G.; TARIQ, S.; TRAVERSA, D.; SCHUSTER, R. 2011. Morphology of the infective larval stage of the equid parasite Habronema muscae (Spirurida: Habronematidae), from houseflies (Musca domestica). Parasitol Res. 108(3): 629-32.

CARDONA, J.; VARGAS, M.; PERDOMO, S. 2014. Frecuencia de Pythiosis cutánea en caballos de producción en explotaciones ganaderas de Córdoba, Colombia. Rev. Med. Vet. Zoot. 61 (I): 31-43.

DOWN, S.; HUGHES, I.; HENSON, F. 2009. Cutaneous habronemiasis in a 9 year-old Arab gelding in the United Kingdom. Equine Vet Educ. 21(1):4-8.

FERREIRA, G.; ALMEIDA, M.; NUENES I. 2013. Casos de Habronemose equina na região do Baixo Jaguaribe-CE. In: Revista de Educação Continuada em Medicina Veterinária e Zootecnia. p. 74.

FREITAS, A. 2013. Habronemose cutânea Abordagem parasitológica e terapêutica. Dissertação de Mestrado em Medicina Veterinária, Universidade de Trás-os-Montes e Alto Douro. Villa Real, Portugal. 83pp

MEHLHORN, H. 2008. Habronema especies. In: Encyclopedia of parasitology [Internet]. p. 1577. Zoomorphology, Cell Biology and Parasitology institute. Düsseldorf, Germany. [Acceso: 15/07/2016]. Disponible en: http://books.google.com.pe/books?id=Jpg1ysgVn-AC

PATERSON, S. 2009. Cutaneous habronemiasis. Equine Vet. Educ. 21(1): 9-10

PUGH, D.; HU, X.; BLAGBURN, B. 2014. Habronemiasis: Biology, Signs, and Diagnosis, and Treatment and Prevention of the Nematodes and Vector Flies. J Equine Vet Sci. 34(2): 241-8.

SCHUSTER, R.; SIVAKUMAR, S.; KINNE, J.; BABIKER, H.; TRAVERSA, D.; BUZZELL, G. 2010. Cutaneous and pulmonal habronemosis transmitted by Musca domestica in a stable in the United Arab Emirates. Vet Parasitol. Elsevier B.V. 174(1-2):170-4.

TRAVERSA, D., R. IORIO, L.; PETRIZZI, I.; DE AMICIS, S.; BRANDT, A.; MEANA, A. 2007. Molecular diagnosis of equid summer sores. veterinary parasitology.150(1): 116-121. 